Service social

\title{
Nouvelles pratiques des organisations populaires... Du militantisme au bénévolat au service de l'État
}

\section{Jean Panet-Raymond}

Volume 34, numéro 2-3, 1985

L’organisation communautaire

URI : https://id.erudit.org/iderudit/706277ar

DOI : https://doi.org/10.7202/706277ar

Aller au sommaire du numéro

Éditeur(s)

École de service social de l'Université Laval

ISSN

1708-1734 (numérique)

Découvrir la revue

Citer cet article

Panet-Raymond, J. (1985). Nouvelles pratiques des organisations populaires... Du militantisme au bénévolat au service de l'État. Service social, 34(2-3),

340-352. https://doi.org/10.7202/706277ar d'utilisation que vous pouvez consulter en ligne. 


\section{Nouvelles pratiques des organisations populaires... Du militantisme au bénévolat au service de l'État}

\section{Jean Panet-Raymond}

La crise économique actuelle bouleverse la structure du capital, les classes sociales, l'État et même certaines habitudes ou valeurs, tel le travail salarié régulier. Elle bouleverse aussi les organisations syndicales et populaires. Si l'on n'ose pas parler de crise chez celles-ci, on peut tout au moins parler d'une nouvelle réalité et d'un climat idéologique qui doit soulever un sérieux questionnement et une réévaluation de la conjoncture et des pratiques. Que doivent faire les organisations devant cette nouvelle réalité et surtout ces nouvelles règles imposées par l'État, cet État-Providence tant décrié mais duquel les organisations dépendent beaucoup (trop?) financièrement? Le discours politique des gouvernements fédéral et provincial est à la fois attirant et contraignant, voire opprimant. Il y a de nouveaux enjeux importants pour la survie des organisations populaires : les groupes suivront-ils la voie du militantisme critique et politique ou la voie du bénévolat au service (au secours?) de l'État?

C'est là le débat que nous voulons soulever en tentant d'identifier quelques nouvelles pratiques des organisations populaires, issues ou entraînées par des facteurs externes (conjoncturels) et internes aux organisations, que nous définirons, d'entrée de jeu, afin de mieux saisir les pratiques elles-mêmes.

\section{Les facteurs externes aux organisations (conjoncture)}

La crise économique a créé des conditions extrêmement difficiles, objectivement, par la hausse de l'inflation et des taux d'intérêt, et surtout par le chômage, provoqué par les mises à pied et la faible 
création d'emplois. On retrouve donc deux "demi-générations" de jeunes (moins de 30 ans) et de plus âgés (plus de 45 ans) forcées de ne plus penser à une retraite après une vie de travail rémunéré, ou forcées de prendre une retraite anticipée et donc souvent mal préparée. Le drame humain est aussi pénible. Mais plus qu'un gonflement des rangs de chômeurs et assistés sociaux, la crise a créé un climat d'insécurité et de perte d'illusion, voire de désespoir, face à un avenir meilleur. On perçoit que les règles du jeu sont changées : l'emploi régulier comme solution n'existe à peu près plus. La précarisation de l'emploi a frappé plus particulièrement les jeunes mais aussi les femmes, qui avaient commencé à accéder en plus grand nombre au marché du travail. Cela a forcé plusieurs personnes à réajuster leurs ambitions et leurs projets. Deux voies semblent se dessiner: soit que l'on développe des formes de travail au noir, le plus souvent individualisées, avec l'aide de réseaux amicaux et familiaux, ${ }^{1}$ soit que l'on tente d'obtenir du travail à contrat ou dans des programmes de création d'emplois pour les jeunes. Ou alors on tente de s'insérer dans des organismes communautaires et populaires pour éventuellement y décrocher un emploi. C'est une voie qui influencera évidemment les organisations populaires.

L'accroissement des problèmes économiques se traduit évidemment aussi par un accroissement des besoins sociaux, alors que les revenus de l'État diminuent. II en résulte donc une crise ${ }^{2}$ qui se répercute fortement sur les organisations populaires. Alors que le début des années 1970 a été marqué par des revendications à l'égard de l'État, les années 1980 se caractérisent par de nouveaux rapports avec celui-ci. D'une part, le gouvernement du Parti québécois a créé de nombreux appareils revendiqués par les groupes (Régie du logement, Office de services de garde, Office des handicapés, Conseil du statut de la femme, Secrétariat à la jeunesse, etc.); d'autre part, il a manifesté la volonté de réduire son intervention dans le champ social. Les organisations sont un peu déséquilibrées: les appareils d'État revendiqués existent, sans pour autant répondre aux attentes. La bureaucratisation et la technocratisation ${ }^{3}$ des services ont mêlé les cartes: il est certes plus facile de lutter pour l'obtention d'un droit, d'une loi ou d'une ressource que de lutter pour que cette loi ou ressource réponde aux attentes. Les groupes sont donc un peu piégés dans les champs déjà labourés et doivent innover! Mais l'État, lui, a " innové" avec son discours sur la concertation (le partenariat) avec les organismes du milieu, la prise en charge par le milieu, le communautaire, le bénévolat... Poussés par des considérations essentiellement budgétaires, les politiciens et les technocrates tiennent un nouveau discours : 
"Une des principales priorités de mon gouvernement sera la révision complète des programmes sociaux du gouvernement en vue d'économiser autant d'argent que possible. Une façon d'atteindre cet objectif consiste à encourager le secteur du bénévolat à participer davantage à la réalisation de nos programmes sociaux [...] Le secteur du bénévolat au Canada représente une ressource inutilisée qui peut réduire les dépenses du gouvernement, mais peut aussi créer des emplois en même temps [...] Le bénévolat représente aujourd'hui la méthode la plus rentable de fonctionnement au Canada [...]" 4

"Les "individus ordinaires" ont davantage de potentiel, d'habiletés et d'intérêt pour s'aider et se supporter les uns les autres, que ne le supposait l'approche de l'État-Providence [...] Les ressources spécialisées des intervenants sociaux demeureront nécessaires, mais les politiques d'intervention, devront d'abord et avant tout viser à équiper et supporter les réseaux informels, lorsqu'ils existent, à les créer ou les développer lorsque inexistants ou trop faibles. ${ }^{5}$

"Lorsque ces ressources [des réseaux primaires et communautaires] seront impuissantes à répondre aux besoins exprimés, le réseau public de services socio-communautaires devra être mis à contribution $[\ldots]{ }^{6}$

Et voilà : le message est lancé, de Reagan à Mulroney, en passant par Thatcher et le Rapport Barclay: ${ }^{7}$ il faut couper et il faut établir un partenariat avec les groupes. Mais lesquels et à quelles conditions? Voilà l'enjeu important qui est déterminant pour les organisations populaires: est-ce enfin l'heure de gloire des groupes populaires qui acquièrent la reconnaissance tant réclamée ou est-ce une forme de récupération d'acquis comme on a connu au début de la réforme Castonguay ? ${ }^{8}$ L'État tient le gros bout du bâton, dans ce partenariat : le financement. Or, on connaît par expérience la tendance "naturelle " de l'État à contrôler ce qu'il finance, surtout lorsqu'il s'agit d'activités qui peuvent représenter un ferment de contestation sociale et politique.

Les groupes vivent déjà les contraintes de la dépendance à l'égard de l'État. ${ }^{9}$ La bureaucratisation de l'appareil, la professionnalisation des interventions et la segmentation des clientèles imposées par la programmation de l'État amènent les groupes à adopter les mêmes comportements. Voilà une belle contradiction: les groupes développent justement des pratiques que l'État dit vouloir modifier. ${ }^{10}$ Donc, dans les faits, l'État force les groupes à définir leurs priorités en fonction des définitions de besoins déterminés par les programmescadres. Est-ce un hasard si l'on voit naître une prolifération de garderies, maisons de femmes violentées, maisons de jeunes, services de maintien à domicile? Les groupes d'alphabétisation, les associations 
de locataires, les ACEF ont-ils modifié leur pratique pour garder leur financement?

À ce niveau, peut-on vraiment parler de reconnaissance des groupes communautaires et populaires? Est-ce que ce partenariat se fait dans le respect de l'autonomie des organisations? La récupération des groupes est-elle imminente? Ces questions méritent réflexion. Le choix est-il tranché entre le bénévolat au service de l'État et le militantisme autonome, "pur et dur"? Les groupes doivent saisir l'occasion de se démarquer par des pratiques innovatrices et autonomes.

Dans ce dilemme difficile des groupes face à cette politique, le mouvement syndical aurait pu offrir un soutien important. Or, la " crise" du syndicalisme québécois est un autre facteur important dans la pratique des organisations populaires; il est lui aussi piégé par la crise économique et celle de l'État. Le gel relatif des effectifs ${ }^{11}$ des secteurs public et para-public crée une stagnation de la syndicalisation, alors que la privatisation de plusieurs services a déjoué les règles de l'emploi par l'utilisation de contractuels. La "précarisation" de l'emploi a aussi créé une armée d'emplois temporaires, "sur appel" et surnuméraires, souvent non syndicables. Parallèlement, les mises à pied dans plusieurs entreprises ont aussi diminué les effectifs syndiqués, alors que les nouvelles entreprises se prémunissent de plusieurs façons pour empêcher la syndicalisation.

Les difficultés internes, entraînées notamment par des négociations plus difficiles et une perte de membership, ont aussi affaibli le leadership du mouvement syndical. Il y a une recherche d'orientation politique, qui oscille entre le corporatisme syndical et des initiatives comme le Fonds de solidarité de la F.T.Q., (appuyé par les gouvernements fédéral et provincial).

On sent un flottement dans les Centrales, qui ont de la difficulté à ajuster leur message sur la crise, les jeunes, les femmes et les conditions de vie hors-travail. La perte de leadership dans ces domaines se manifeste, tant au plan national que régional, et dans les sommets populaires qui piétinent. Le mouvement syndical doit se redéfinir ${ }^{12} \mathrm{car}$ il n'exerce plus le rôle actif qu'il a déjà exercé dans le champ des conditions de vie, en collaboration avec les groupes populaires.

Un dernier facteur conjoncturel, qui rejoint presque les facteurs internes, est l'apparition, depuis quelques années, de "nouvelles clientèles" et de nouvelles préoccupations qui ont un impact sur les revendications et les pratiques des organisations populaires. Mais, en fait, ces clientèles sont aussi le produit d'une segmentation de la population par les programmes gouvernementaux. On pense ici surtout 
aux jeunes, aux sans-emploi, aux femmes violentées, aux personnes âgées. Cela a donné naissance à plusieurs organismes qui poussent comme des champignons (et qui meurent parfois comme des éphémères !): maisons de jeunes, organismes de loisirs et d'animation, maisons d'hébergement pour jeunes en difficulté et pour femmes violentées, centres de femmes, centres de recherche et de préparation à l'emploi, centres d'accueil pour immigrants et réfugiés, etc. On ne peut s'empêcher de penser aux programmes de financement, voire même aux contrats de services qui permettent leur survie.

À travers ces organismes "de services", on retrouve aussi des initiatives à caractère économique - beaucoup en milieu rural - qui tentent de répondre à des fermetures d'usine ou de chantier. Ces initiatives locales à petite échelle ont aussi développé des rapports différents à l'État qui influencent les organisations populaires dans leur ensemble.

Enfin, on pourrait ajouter, en marge de ce facteur, une influence issue des courants professionnels de l'intervention sociale qui parlent d'approches communautaires, ${ }^{13}$ écologiques, ${ }^{14}$ systémiques, ${ }^{15}$ structurelles ${ }^{16}$ et de réseaux. ${ }^{17}$ Toutes ces approches développent plus ou moins le besoin de parler des dynamismes locaux et informels et de bâtir des groupes et des actions collectives à partir des besoins individualisés.

\section{Les facteurs internes aux groupes}

Plusieurs des facteurs internes étant corollaires aux facteurs externes, nous n'élaborerons pas très longuement sur ceux-ci.

Les difficultés de financement ont, à la fois exigé plus d'énergie pour obtenir moins d'argent et de façon moins stable, et ont limité les ressources humaines et matérielles des groupes dans l'ensemble. Cette situation aurait pu entraîner une dépendance plus grande à l'égard des membres, militants ou bénévoles, et mener à des luttes de mobilisation plutôt que des services exigeants en temps et en argent. Mais, on constate plutôt un repli sur le quotidien dans la vie organisationnelle. On a de la difficulté à faire les grandes mobilisations de jadis, soit pour défendre des revendications, soit pour mener des campagnes d'autofinancement.

Le financement est donc devenu un carcan accaparant et étouffant qui impose parfois une redéfinition des champs d'action des projets en fonction des subventions possibles. ${ }^{18}$ Des groupes doivent même remettre en question leur autonomie et leur mode de fonctionnement afin de recevoir certaines subventions. ${ }^{19}$ Le cas des associations de 
locataires à Montréal n'est qu'un triste exemple du resserrement du financement qui fait plier des organisations acculées à la faillite. ${ }^{20}$

Ces problèmes ont aussi entraîné, chez les groupes, une technocratisation et une spécialisation, voire une professionnalisation, qui exigent de telles compétences que l'on exclut les militants "ordinaires» des postes de permanents et des postes électifs. La vague populiste a été de courte durée et les militants plus formés, pour ne pas dire les intellectuels, ont repris le contrôle des organisations.

Cela a mené à un autre phénomène, interne aux organisations : la bureaucratisation et l'institutionnalisation. On se donne des règles assez étroites et on les reproduit. Cela peut devenir sclérosant si le "mimétisme institutionnel» ne laisse pas place aux discussions et aux saines critiques de nouvelles personnes, membres ou usagers. ${ }^{21}$ On reproduit un certain fonctionnement, sans pour autant réitérer un projet politique et un objectif de changement. Les jeunes qui arrivent dans certaines organisations se voient mal reçus s'ils critiquent sans respecter les règles du jeu. Le "crois ou meurs" est remplacé par le "crois ou quitte». Cette difficulté, chez plusieurs groupes, à être perméable à leur critique se retrouve aussi dans les syndicats. On peut donc comprendre les étiquettes anarchisantes collées aux organismes de jeunes et, par ailleurs, le sentiment d'aliénation qu'ils ressentent face à plusieurs organisations contrôlées par des «vieux» militants.

Ce dernier facteur nous amène à soulever la question du vieillissement et même de l'usure des militants et des permanents. La relève est absente et le contrôle reste aux mains de personnes qui souffrent parfois de burn out. Elles se défendent des conséquences d'un rythme fou par une tentative de retrouver une vie privée personnelle moins collée au militantisme à temps plein. Le dynamisme d'antan cède parfois au bureaucratisme routinier, pour se terminer par un départ mérité et salutaire pour tous. Mais partir, c'est mourir un peu; c'est difficile. C'est d'autant plus difficile que l'on n'a pas toujours préparé la relève ni dans le sens de la continuité (et c'est peut-être bien ainsi) ni dans celui d'un changement fondé sur une bonne analyse de l'organisation et de la nouvelle conjoncture. Ainsi, certains groupes se retrouvent obligés de se rebâtir avec peu de ressources, peu de membres et peu d'énergie devant l'absence de projet politique enthousiasmant et mobilisant.

Dans ce contexte, il faut innover, développer de nouveaux rapports à l'État, de nouvelles pratiques quotidiennes, de nouvelles formes d'organisations et de nouvelles alliances pour rejoindre la population et défendre des droits fondamentaux qui sont menacés. 


\section{Nouvelles pratiques, nouveaux rapports}

C'est encore au niveau d'observations empiriques plus que d'analyses rigoureuses et catégorisées que l'on peut identifier certaines modifications dans les pratiques des organisations populaires. Ce texte n'a donc pas la prétention d'un rapport d'enquête, importante à mener par ailleurs, mais se veut une contribution au débat et à la meilleure compréhension de la situation actuelle et des pistes à choisir. Les observations suivantes sont faites à partir d'expériences personnelles auprès de groupes variés, à Montréal surtout et dans l'ensemble du Québec.

On remarque d'abord un certain déplacement des préoccupations des groupes de l'idéologique à l'économique. De la recherche de la "ligne juste" et du projet politique, on en est plutôt venu à des préoccupations de "pain et beurre», ici et maintenant. Les grands palabres idéologiques sont remplacés par des discussions de "comment faire" pour avoir un impact sur la vie quotidienne actuelle. ${ }^{22}$ Les revendications de plusieurs groupes illustrent bien cette tendance : les associations de locataires réclament des logements sociaux (coopératives et H.L.M.) ; les jeunes du Regroupement autonome des jeunes (R.A.J.) réclament la parité de l'aide sociale pour les moins de trente ans et de vrais emplois; les syndicats et groupes de sans-emploi revendiquent de "vrais jobs" et pas juste du bénévolat; les femmes s'opposent à la vente de documents pornographiques et réclament un soutien financier pour les maisons de femmes violentées; les syndicats et travailleurs non syndiqués luttent pour la survie d'entreprises menacées de fermeture. Ces quelques exemples dénotent, non seulement une volonté de toucher la population dans ses besoins concrets, mais aussi de tenir compte des capacités organisationnelles limitées des groupes. On tente d'arracher des gains précis et réalistes, sans faire la révolution.

Une conséquence de ce genre de revendications est la création de regroupements sur des enjeux précis, à court terme. On se regroupe plus ponctuellement avec des structures plus "légères", le temps nécessaire pour mener la lutte. On parlerait donc plutôt de "coalition" plus large, souvent justement parce que l'objectif est plus étroit, plứs concret, et ce sans faire appel aux orientations idéologiques qui pourraient être antagoniques. On est plus tactique... ou on valorise plus les petites organisations locales, qui se regroupent le temps d'une lutte. Ainsi, on fera alliance avec des groupes de loisir, des groupes d'église et même des politiciens, pour obtenir un changement de zonage (pour limiter les lieux de vente de matériel pornographique), 
pour obtenir des H.L.M., pour modifier le passage d'une autoroute, pour déterminer le lieu d'un éventuel C.L.S.C., pour sauvegarder un bâtiment historique dans un quartier, etc. Les groupes populaires ne jouent plus aux purs et foncent en faisant certains compromis. C'est de cette façon, en élargissant leur "base naturelle", qu'ils réussissent à arracher des gains appréciables.

Les succès du regroupement des jeunes, par le R.A.J., sont liés à sa capacité de rallier rapidement et simplement autour de revendications concrètes. Lorsque les débats idéologiques prendront le dessus, la large base s'effritera, comme dans plusieurs regroupements plus structurés et "lourds": on pense notamment à La Grande marche pour l'emploi, le dernier Front commun du secteur public, les regroupements provinciaux d'Associations de locataires, d'Associations coopératives d'économie familiale (ACEF), de chômeurs et de chômeuses, de garderies, etc. Il faut reconnaître le côté positif de ce nouveau pragmatisme, mais il faut aussi en questionner les dangers à long terme, devant l'absence de perspective politique. On risque de tomber dans un activisme qui accumule les actions ponctuelles, sans tabler sur ces acquis pour modifier les rapports de pouvoir fondamentaux. Cela rappelle un peu les débuts de l'organisation communautaire d'influence américaine et l'animation sociale urbaine des années soixante.

Une autre pratique, pas vraiment nouvelle, c'est l'importance mise sur le service de qualité, professionnel, compétent et efficace. Si l'on n'oppose plus service et mobilisation, c'est peut-être parce qu'il n'y a plus autant d'intérêt à mener les grandes luttes; on tend plutôt à répondre à des besoins immédiats, concrets et nombreux. C'est aussi, peut-être, parce qu'on entre dans une pratique qui valorise plus le vécu, le privé et l'individu, tout comme le savoir et l'expertise: ne devient pas consultant budgétaire (ACEF) ou permanent d'une association de locataires ou d'un comité de chômeurs qui veut ! Il faut faire un apprentissage juridique et technique assez difficile. On assiste donc à une certaine spécialisation des problématiques, devenues de plus en plus complexes, et ainsi à une professionnalisation et une technocratisation des organisations populaires. Est-ce le résultat de la présence plus grande de diplômés, que le marché régulier de l'emploi n'absorbe pas? ou le résultat des exigences du financement de l'État? En effet, celui-ci dénonce les coûts élevés de la bureaucratisation et l'inefficacité des professionnels et technocrates, tout en imposant ses propres modèles de fonctionnement aux organismes à qui il veut laisser une partie de ses fonctions. L'État tente de restreindre ses dépenses en "donnant à contrat" aux associations, qui doivent se soumettre au modèle technocratique et prendre la relève... mais à moindre coût. La 
contraction est difficile à accepter. II y a certes un certain gain de qualité dans le service offert par les groupes, mais il y a un recul par rapport au rôle de changement structurel que se donnaient la plupart des organisations populaires.

Ces nouvelles pratiques et le contexte du financement restreint ont amené plusieurs groupes à des rapports différents avec l'État. L'affrontement fait tranquillement place à une concertation prudente. Besoin tactique, évolution idéologique ou simple bon sens? Aucune réponse n'est vraiment satisfaisante. II demeure que les tables de concertation regroupant organismes publics et populaires se font de plus en plus nombreuses à l'heu re où l'État parle de concertation et de prise en charge par le milieu, voire même de décentralisation des ressources publiques. ${ }^{23}$ Les liens entre organismes différents d'un même milieu géographique (quartier, ville) semblent parfois plus importants que les liens entre les organismes ouvrant dans le même domaine. On crée des réseaux d'échange et même d'influence, mais localement ; c'est peut-être un réseau plus "naturel" ? II n'est certainement pas toujours facile à mobiliser, si ce n'est que ponctuellement ou sur des échanges de services très concrets. Nombreuses sont les tables de concertation, sectorielle ou générale, qui sont inactives. Mais, devant ce nouveau localisme axé sur les petites structures, on peut se demander si l'on ne tend pas à la création de "petites patries" ou de comités de quartier dans l'optique d'un contrôle communautaire. On a parfois l'impression, en regardant notamment le travail des nouveaux C.L.S.C., de revenir au modèle de développement communautaire américain ou aux velléités du mouvement du community control. ${ }^{24}$ On favorise donc des rapports harmonieux entre les différents partenaires (État, organisations populaires, organismes du milieu) en vue de créer des ressources locales non gouvernementales.

Une autre pratique qui découle de cette recherche de modèle léger de gestion est la tendance vers l'autogestion. On délaisse le modèle de démocratie élective (assemblée générale de membres, conseil d'administration) en faveur de différentes formes d'autogestion par les permanents et les militants impliqués quotidiennement. C'est peut-être là une forme plus efficace et la reconnaissance formelle d'une situation de fait : les permanents ont presque toujours eu une influence marquante sur les décisions prises par les instances élues des groupes. Donc, au lieu de se leurrer dans le modèle communautaire "traditionnel", on fonctionne avec les personnes présentes. C'est la consécration du principe de la légitimation de la participation active. Car, sans cela, les membres ou usagers n'ont aucun pouvoir dans l'organisation. C'est la participation très active (et très connaissante) 
qui donne droit au chapitre et non le simple statut, jadis suffisant pour faire valoir (théoriquement et parfois pratiquement) ses idées. Avec cette tendance, on a l'impression que les groupes abandonnent un peu le concept d'organisation de masse, et on ne s'efforce plus à tout prix de "faire participer" les membres. On reconnaît la situation réelle et on avance avec les personnes actives. Le membership devient une ressource à laquelle on fait appel de façon très ponctuelle. C'est encore une conséquence du modèle technocratique de fonctionnement. Les garderies, notamment, semblent avoir adapté ce mode de gestion depuis leur financement public plus stable, par le biais de l'Office des services de garde. La complexification du financement et des tâches, l'arrivée de permanents plus diplômés, la syndicalisation des employés; tous ces facteurs ont contribué à évincer les parents de la position d'usagers impliqués et donc ayant un certain pouvoir décisionnel. ${ }^{25}$

Lié à la disparition de ce souci des membres participants, on constate que le rythme effréné a diminué sans pour autant cesser complètement. Le militantisme à temps plein semble mourir au profit d'une "semaine à peu près normale" qui permet de reprendre son temps passé en réunion les soirs et les fins de semaine. On ne planifie plus la révolution pour le lendemain! On laisse place à des projets personnels et à une vie de couple. C'est la revanche de la vie privée qui reprend sa place, alors même que les revendications de plusieurs groupes de femmes ont justement rendu publics et politiques plusieurs aspects de cette vie privée.

Enfin, une pratique aussi liée à la précédente est la tendance à organiser, dans les groupes, des activités plus socio-culturelles: fêtes, repas communautaires, films-causeries. Le style austère et ascétique des organisations militantes laisse sa place à un courant d'air frais. On prend le temps de faire des activités plus agréables pour les membres, mais aussi permettant de rejoindre la population en général. Les groupes de femmes et de jeunes et les Associations pour la défense des droits sociaux (A.D.D.S.) ont ouvert la voie à ces activités où l'on se rencontre dans un contexte plus facile. "C'est un moyen de rejoindre notre monde, pis c'est le fun". Cette déclaration d'un jeune vivant de l'aide sociale aurait pu être complétée par: "pis ça permet même de mieux faire passer notre message ". On n'organise donc pas seulement une fête après la lutte gagnée (ou perdue), on organise la fête pour gagner la lutte. C'est là la nouveauté de la pratique. Les groupes seraient-ils sortis des ténèbres pour arriver à la société de loisirs tant annoncée? 


\section{Conclusion}

Sans pouvoir parler d'un renouveau, on peut déceler certaines pratiques nouvelles qui sont encourageantes dans la mesure où elles peuvent témoigner d'une réalité parfois trop figée dans sa façon d'être et de faire. Les organisations populaires doivent être, par définition même, souples et capables de répondre aux besoins de la vie, qui évoluent et changent. Ce n'est pas l'institutionnalisation qui pourra donner une force à ces organisations mais plutôt sa capacité d'adaptation et sa capacité de réagir rapidement aux événements.

Mais, si les groupes doivent évoluer, ils ne doivent pas pour autant oublier leur vocation première de changement structurel en faveur des intérêts des classes populaires. Il est donc important de faire une sérieuse analyse de la situation et une auto-critique qui permettra de prendre position face aux enjeux actuels. Devant les "avances" de l'État, il est essentiel de maintenir des solidarités et de créer des alliances, en sortant de l'isolement du localisme (plus facile), pour se développer en favorisant l'autonomie des organisations populaires dans ce partenariat presque inévitable. Le défi sera de collaborer à des projets avec l'État (notamment par le biais des C.L.S.C.) sans être totalement récupéré au point de perdre de vue les objectifs de changement pour des rapports plus égalitaires.

\section{Notes et références}

* Je tiens à remercier Denyse Côté qui a soutenu la production de ce texte en fouillant et confrontant mes idées.

1 Charbonneau, F., J. Panet-Raymond et C. Polrier, Les conditions de vie des salariés québécois et leur stratégie, en temps de crise, rapport de recherche, École de service social, Université de Montréal, mars 1985.

2 Ouellet, Hector, Les services sociaux communautaires: quelques éléments bibliographiques, Montréal, Fédération des centres locaux de services communautaires du Québec (F.C.L.S.C.Q.), avril 1984. Pierre Rosanvallon, La crise de l'Etat-Providence, Paris, Seuil, 1981.

3 Nous avons utilisé ces deux termes selon les sens donnés par Jacques GodBout, dans: La participation contre la démocratie, Montréal, SaintMartin, 1983, p. 153. - La bureaucratie c'est «le fait que des membres de l'administration publique exercent un pouvoir qui repose sur la connaissance et l'application des règles de fonctionnement créées par eux et qu'ils sont seuls à contrôler [...]" - "La technocratie, c'est aussi l'exercice d'un pouvoir, qui repose cette fois sur une connaissance d'expert dans un domaine et non pas sur la connaissance des règles de fonctionnement de l'organisation». 
4 Mulroney, Brian, discours prononcé lors d'un atelier sur la politique sociale au Congrès du Parti conservateur, le 10 juin 1984 à Ottawa, et cité dans I'ICEA, bulletin de liaison de l'Institut canadien d'éducation des adultes, vol. 6 , n० 4-5, 1984, p. 3.

5 Ouellet, H., Les services sociaux au Québec: éléments de réflexion pour les années 1980, Montréal, F.C.L.S.C.Q., octobre 1983, p. 10.

6 Ministère des affaires sociales, Le partage des responsabilités C.S.S.-C.L.S.C. en matière de services sociaux, Québec, décembre 1983, p. 25.

7 Barclay, Peter, Social Workers, Their Roles and Tasks, London, Bedford Square Press, 1982. Le "rapport Barclay" sera suivi, en 1985, par le rapport Fowler qui doit réévaluer plus spécifiquement le système des services sociaux. Le gouvernement Thatcher poursuit son évaluation pour diminuer son intervention. L'influence actuelle des politiques restrictives anglaises semble comparable à l'influence qui a donné naissance à la réforme québécoise de 1971 suite au Rapport Seebohm (1968) qui prônait la création de services sociaux et de santé polyvalents et décentralisés.

8 Voir, à ce sujet: J. Godbout et N. Martin, Participation et innovation, Montréal, I.N.R.S.-ÉNAP, 1974, (rapport de recherche); et aussi : J. GODBOUT et J.-P. ColuIN, Les organismes populaires en milieu urbain; contre-pouvoir ou nouvelle pratique professionnelle?, Montréal, I.N.R.S.-Urbanisation, 1977. ("Rapport de recherche", 3.)

9 HAMEL, Pierre, "Crise de la redistribution étatique et financement des organisations populaires", Revue internationale d'action communautaire, 10/50, automne $1983: 63-76$.

10 VaILLANT, Jeanne-d'Arc, La pratique sociale de demain au Québec, allocution d'ouverture au Congrès interrégional de la Corporation des travailleurs sociaux du Québec, Québec, octobre 1983, 25p.

11 Bıouin, Jean, "Les camouflés, les invisibles et les disparus", L'Actualité, mai $1985: 45-53$.

12 Saint-Pierre, Céline, "Le difficile chemin du mouvement syndical", Mouvements, mai $1985: 10-11$.

13 Plusieurs textes issus du Centre des services sociaux du Montréal métropolitain et du Ministère des affaires sociales illustrent cette tendance ainsi que quelques textes de la F.C.L.S.C.Q.

${ }^{14}$ Camil Bouchard et Claire Chamberland ont bien illustré cette approche. On peut lire notamment : C. BOUCHARD, "Apprendre la psychothérapie", dans : Psychothérapie : attention, Québec, Québec-Science, 1983.

${ }^{15} \mathrm{Ce}$ courant est probablement celui qui se développe le plus au Québec actuellement, et notamment dans des organismes tels les Alcooliques anonymes, Parents anonymes, Al-Anon, etc. On peut lire: J. GUAY, "Les aidants non professionnels", dans: Psychothérapie: attention!, Québec, Québec-Science, 1983.

16 Ce courant est enseigné dans les universités et utilisé dans certains groupes de service plus politiques. M. MOREAU, "L'approche structurelle familiale en service social", Revue internationale d'action communautaire, 7/47, printemps 1982: 159-172.

17 Bien qu'il y ait plusieurs tendances à l'intérieur de l'approche réseau, on peut identifier l'équipe qui a poussé le plus la recherche dans le domaine: Claude 
Brodeur, Richard Rousseau et al., L'intervention de réseaux, Montréal, France-Amérique, 1984.

18 Ror, Louis, "Le repli forcé du mouvement populaire", Mouvements, mai $1985: 12-13$.

19 HaMel, P., op. cit.

20 BOUCHer, L. et al., La coalition charitable : bilan d'une lutte, mars 1984, texte polycopié. On y relate la lutte des associations de locataires contre CentraideMontréal, qui exigeait l'obtention du numéro d'organisme charitable de la part des associations, sous menace de devoir couper leur financement.

21 PANet-RAYMond, J., L'absence de mémoire collective et le mimétisme institutionnel dans les organisations populaires ou comment on oublie ce qu'on a fait en continuant à faire la même chose!, allocution prononcée au colloque sur La continuité dans les organisations populaires, 16 octobre 1982. Publié dans le rapport du Colloque par l'Institut canadien d'éducation des adultes.

22 On remarque, à cet effet, un certain nombre de textes-outils sur le financement, l'utilisation des médias, l'animation de groupe, l'intervention communautaire, qui remplacent ceux sur les grands débats idéologiques.

23 Rapport Barclay.

24 Altshuler, Alan, Community Control, New York, Pegasus, 1970.

25 COTE, Denyse, Du maternage privé au maternage public... ou de la mère couveuse à la mère coureuse, Université de Montréal, 1984, (texte polycopié). 